\title{
A constant phase approach for the frequency response of stochastic linear oscillators
}

\author{
Emmanuelle Sarrouy ${ }^{1, a}$, Emmanuel Pagnacco ${ }^{2}$ and Eduardo Souza de Cursi ${ }^{2}$ \\ 1 LMA, Centrale Marseille, CNRS, UPR 7051, Aix-Marseille University, 13451 Marseille, France \\ 2 LOFiMS, INSA de Rouen, BP 8, 76801 Saint-Étienne du Rouvray, France
}

Received 29 October 2014, Accepted 10 May 2015

\begin{abstract}
When studying a mechanical structure, evaluation of its frequency response function (FRF) over a given frequency range is one of the main interests. Computational cost aside, evaluating FRFs presents no methodological difficulty in the deterministic case. Doing this when the model includes some uncertain parameters may however be more difficult as multimodality and discontinuity can arise around resonances. Indeed, even for a single degree of freedom system, it can be shown that usual methods of the probabilistic frame such as generalized Polynomial Chaos may fail to properly describe the probability density function of the response amplitude. This study proposes another approach which involves a shift in the usual quantities used to draw FRFs. Instead of computing the stochastic response for a given excitation frequency, this work adopts a constant response phase point of view. For each phase value of the oscillator response, the uncertainty over some parameters is propagated to the corresponding uncertain amplitudes and excitation frequencies. This provides much smoother variations of the involved quantities which are much easier to describe using a simple Polynomial Chaos approach. Both analytical and numerical results will be exposed for a single degree of freedom oscillator whose stiffness follows a uniform law.
\end{abstract}

Key words: Structural dynamics / frequency response function / random vibrations / uncertainty propagation / polynomial chaos

\section{Introduction}

When studying a mechanical structure, evaluation of its frequency response function (FRF) over a given frequency range is one of the main interests. Computational cost aside, evaluating FRFs presents no methodological difficulty in the deterministic case. Doing this when the model includes some uncertain parameters may however be more difficult. Analytic expressions can be established for small systems: Udwadia [1,2] provided explicit expressions of the statistics of the response of a single degree of freedom system for random mass, stiffness and damping rate. Heinkelé et al. [3] wrote the explicit formula for the probability density fonction of the FRF of a single degree of freedom oscillator with random damping, either viscous or hysteretic. Expressions for envelopes (confidence intervals) could then be derived and finally lead to a mean to identify damping random properties from experiments. Similar work was conducted for a random stiffness [4-6] showing that multimodality can arise around resonances.

\footnotetext{
${ }^{a}$ Corresponding author:

emmanuelle.sarrouy@centrale-marseille.fr
}

When large or nonlinear systems are involved, numerical methods must be considered as full analytic expressions cannot be established anymore. Since Wiener [7] who proposed the Polynomial Chaos decomposition for Gaussian processes, many developpments were done to enlarge the application of this idea. Winterstein $[8,9]$ introduced the moment-based Hermite model for random vibrations and fatigue. In these works, the polynomial expansion is also generalized to arbitrary polynomials through a procedure analogous to the Gram-Schmidt method. This enables a choice for the represention basis. For instance, Laguerre polynomials have been proposed for positive random process, associated to chi-square- 2 variables. Ghanem and Spanos [10] proposed to combine the polynomial chaos expansion viewed as a generalized Fourier expansion with the finite element method and Xiu and Karniadakis [11] extended this approach by introducing a larger family of polynomials adapted to different distributions, leading to the so-called generalized Polynomial Chaos (gPC; see Ernst et al. [12] for detailed results on convergence). In the last decade, several papers addressed the discretization of the stochastic space to process random processes with a discontinuous dependency to the input random 


\section{Nomenclature}

\begin{tabular}{|ll|}
\hline$a$ & System response amplitude $[\mathrm{m}]$ \\
$f_{0}$ & Modal excitation amplitude $\left[\mathrm{N} \cdot \mathrm{kg}^{-1}\right]$ \\
$\mathcal{I}\left(\Omega_{0}^{2}\right)$ & Squared eigen circular frequency variation range \\
$p_{X}$ & Probability density function of $X$ \\
$\mathbf{P}_{X}$ & Cumulative distribution function of $X$ \\
$\mathbb{P}$ & Probability measure \\
$q$ & System dynamic response (displacement) $[\mathrm{m}]$ \\
$\dot{q}$ & Velocity ( $q$ derivative with respect to time) $\left[\mathrm{m} . \mathrm{s}^{-1}\right]$ \\
$\ddot{q}$ & Acceleration $\left(\dot{q}\right.$ derivative with respect to time) $\left[\mathrm{m} . \mathrm{s}^{-2}\right]$ \\
$X$ & Random variable associated to deterministic variable $x$ \\
$\varphi$ & System response phase [rad] \\
$\eta$ & System damping ratio $[\emptyset]$ \\
$\omega$ & Excitation circular frequency $\left[\mathrm{rad} . \mathrm{s}^{-1}\right]$ \\
$\omega_{0}$ & Eigen circular frequency $\left[\mathrm{rad} . \mathrm{s}^{-1}\right]$ \\
$\omega_{0}^{2}$ & Mean value of squared eigen circular frequency $\left[\mathrm{rad}^{2} . \mathrm{s}^{-2}\right]$ \\
$\Delta \omega_{0}^{2}$ & Half width of $\mathcal{I}\left(\Omega_{0}^{2}\right)\left[\mathrm{rad}^{2} . \mathrm{s}^{-2}\right]$ \\
\hline
\end{tabular}

variables (see Wan and Karniadakis [13,14] for the MultiElement generalized Polynomial Chaos and Foo et al. [15] for the Multi-Element Probabilistic Collocation Method).

Recent work by the authors [16] showed that for a single degree of freedom system, generalized Polynomial Chaos may fail to properly describe the probability density function of the response amplitude. More complex methods such as Multi-Element generalized Polynomial Chaos must be used to address this problem, increasing the computational cost in return.

This study proposes another approach to handle the frequency study of stochastic linear systems. It involves a shift in the usual quantities used to draw FRFs: instead of computing the stochastic response for a given excitation frequency, this work adopts a constant phase point of view. For each phase value of the oscillator response, the uncertainty over some parameters is propagated to the corresponding uncertain amplitudes and excitation frequencies.

This work will be illustrated by a simple single degree of freedom (sdof) linear damped oscillator whose eigen frequency follows a uniform law. This system described in Section 2. Section 3 illustrates multimodality of system response amplitude when the response is sought for a given excitation frequency but variable (free) response phase. Section 4 then develops the proposed approach: the response is sought for a given phase but variable excitation frequency. Section 4.1 provides the equations while Section 4.2 illustrates the approach on the sdof system. Finally, Section 5 proposes a comparison of both methods efficiency - constant excitation frequency and constant phase - when combined to a Polynomial Chaos Expansion.

\section{Stochastic system studied}

\subsection{Deterministic single degree of freedom oscillator}

Let us consider the sdof damped oscillator undergoing a harmonic load depicted in Figure 1. Its movement is

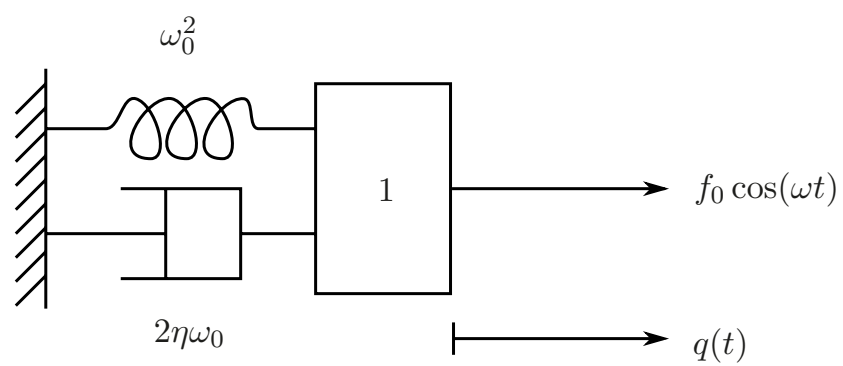

Fig. 1. Simple damped oscillator.

governed by Equation (1).

$$
\ddot{q}+2 \eta \omega_{0} \dot{q}+\omega_{0}^{2} q=f_{0} \cos (\omega t)
$$

where $q$ is the mass displacement, $\dot{q}$ and $\ddot{q}$ are its velocity and acceleration respectively, $\omega_{0}$ is its eigen circular frequency, $\eta$ is the damping ratio, $f_{0}$ is the excitation amplitude and $\omega$ is the excitation circular frequency.

In the frequency domain, Equation (1) becomes

$$
\left(\omega_{0}^{2}-\omega^{2}+2 \mathrm{j} \eta \omega_{0} \omega\right) \hat{q}=f_{0}
$$

where $\mathrm{j}^{2}=-1$ and $\hat{q}$ is the complex amplitude of $q$ :

$$
q(t)=\operatorname{Re}\left(\hat{q} \mathrm{e}^{\mathrm{j} \omega t}\right)
$$

Using these complex notations, one can easily write the complex solution $\hat{q}$ as a function of the mechanical parameters $\omega_{0}, \eta$ and $f_{0}$ and the excitation frequency $\omega$ :

$$
\hat{q}=\frac{f_{0}}{\omega_{0}^{2}-\omega^{2}+2 \mathrm{j} \eta \omega_{0} \omega}
$$

Finally, decomposing the complex quantity $\hat{q}$ into its amplitude $a$ and phase $\varphi$,

$$
\hat{q}=a \mathrm{e}^{\mathrm{j} \varphi}
$$


(a) Amplitude

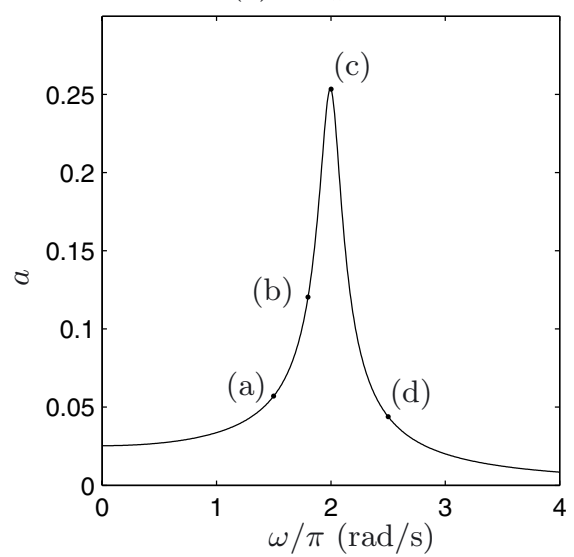

(b) Phase

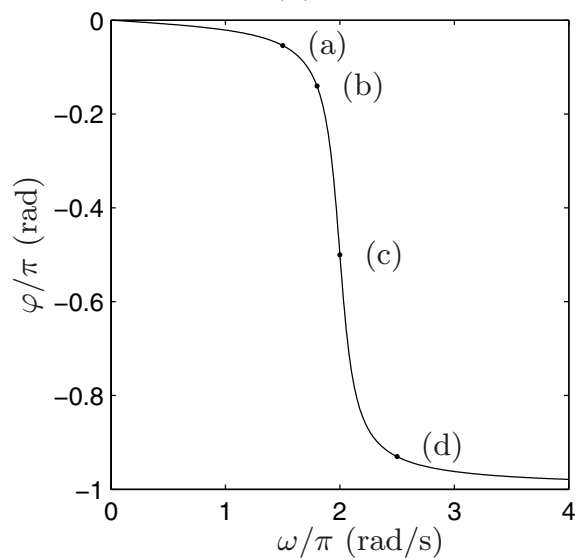

Fig. 2. Deterministic frequency response diagrams for the sdof damped oscillator.

one gets separate expressions for each component:

$$
\begin{aligned}
a & =\frac{f_{0}}{\sqrt{\left(\omega_{0}^{2}-\omega^{2}\right)^{2}+\left(2 \eta \omega_{0} \omega\right)^{2}}} \\
\varphi & =\arctan \left(-\frac{2 \eta \omega_{0} \omega}{\omega_{0}^{2}-\omega^{2}}\right)
\end{aligned}
$$

Amplitude and phase response of the oscillator are displayed in Figure 2 for $f_{0}=1, \omega_{0}=2 \pi$ and $\eta=0.05$.

\subsection{Stochastic single degree of freedom oscillator}

Let us now consider a probability space $(\Theta, \mathcal{A}, \mathbb{P})$ with $\Theta$ the event space, $\mathcal{A}$ the $\sigma$-algebra on $\Theta$, and $\mathbb{P}$ a probability measure. Random variables will be denoted by the capital letter which matches the deterministic variable. Hence if $x$ is a deterministic variable, the associated random variable will be denoted $X$. Its cumulative distribution function (cdf) and probability density function (pdf) will be denoted $\mathbf{P}_{X}(x)=\mathbb{P}(X \leq x)$ and $p_{X}(x)=\frac{\mathrm{d} \mathbf{P}_{X}}{\mathrm{~d} x}$ respectively.

We assume that $\omega_{0}^{2}$ varies and can be modeled using a random variable $\Omega_{0}^{2}(\theta): \Theta \rightarrow \mathbb{R}$ which follows a uniform distribution:

$$
\begin{aligned}
& \Omega_{0}^{2} \hookrightarrow \mathcal{U}\left(\overline{\omega_{0}^{2}}-\Delta \omega_{0}^{2} ; \overline{\omega_{0}^{2}}+\Delta \omega_{0}^{2}\right) \\
& \mathbf{P}_{\Omega_{0}^{2}}(x)=\mathbb{P}\left(\Omega_{0}^{2} \leq x\right)=\frac{x-\left(\overline{\omega_{0}^{2}}-\Delta \omega_{0}^{2}\right)}{2 \Delta \omega_{0}^{2}}, x \in \mathcal{I}\left(\Omega_{0}^{2}\right)
\end{aligned}
$$

with $\mathcal{I}\left(\Omega_{0}^{2}\right)=\left[\overline{\omega_{0}^{2}}-\Delta \omega_{0}^{2} ; \overline{\omega_{0}^{2}}+\Delta \omega_{0}^{2}\right]$.

This may happen when the oscillator stiffness has bounded variations.

Numerical values for later numerical applications are: $\overline{\omega_{0}^{2}}=(2 \pi)^{2}, \Delta \omega_{0}^{2}=0.3 \overline{\omega_{0}^{2}}, \eta=0.05$ and $f_{0}=1$. Diagrams in Figure 2 then match the mean sdof system response over the frequency range $[0 ; 4 \pi]$. Four operating points around which the stochastic response will be detailed are marked in Figure 2 using letters (a) to (d).

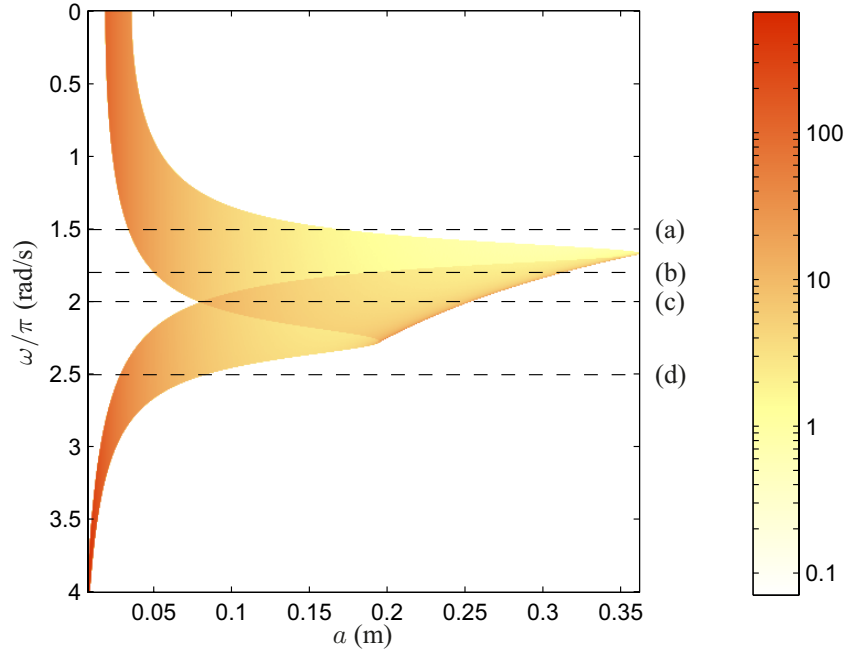

Fig. 3. Constant excitation frequency study: $A$ pdf $\left(p_{A}\right)$ over a given excitation frequency range. Color scale maps $\lg \left(p_{A}\right)$. (a)-(d) cuts refer to operating points defined in Figure 2.

\section{Problems arising when considering a constant excitation frequency}

When studying a linear system over a given frequency range, it is natural to observe the variation of $a$ for a set of excitation frequencies $\omega$. That is what is usually done when considering deterministic structures: for several values of $\omega$, amplitude $a$ and phase $\varphi$ are evaluated and plotted on graphics similar to Figure 2. It then seems natural to use a similar procedure when studying stochastic linear structures over a given excitation frequency range: for several values of $\omega$, a sample of $\Omega_{0}^{2}$ realizations is generated and corresponding realizations of $A$ and $\Phi$ are evaluated. The problem is that, for some values of $\omega$, the probability density function of $A$ is discontinuous as demonstrated by Pagnacco et al. $[6,16]$. This is illustrated in Figures 3 and 4 .

These Monte Carlo simulations are obtained by considering $501 \omega$ values equally distributed over the 
(a) $\omega / \pi=1.50 \mathrm{rad} / \mathrm{s}$

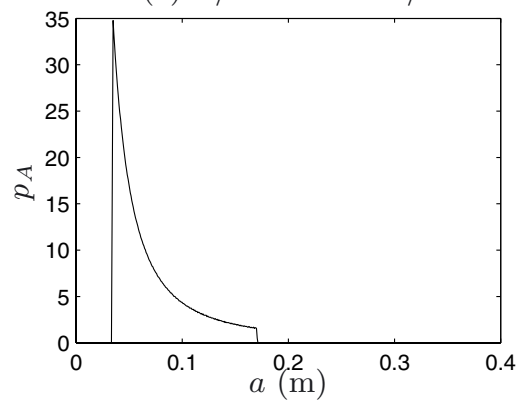

(c) $\omega / \pi=2.00 \mathrm{rad} / \mathrm{s}$

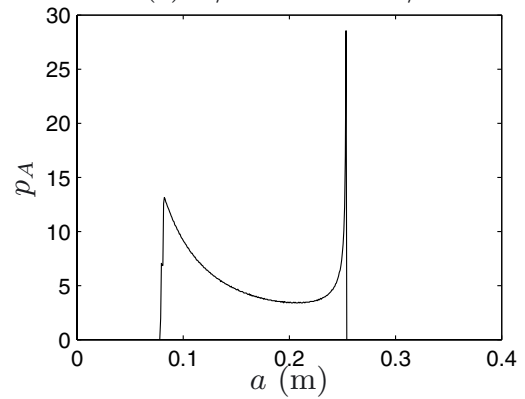

(b) $\omega / \pi=1.80 \mathrm{rad} / \mathrm{s}$

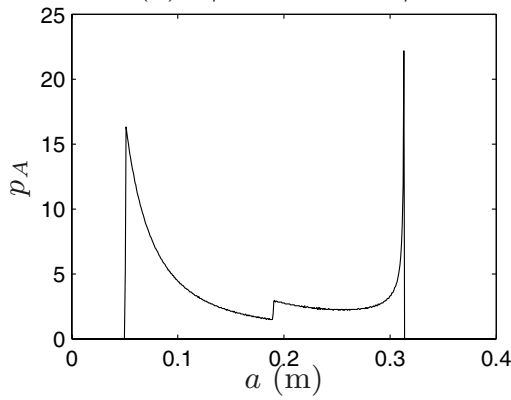

(d) $\omega / \pi=2.50 \mathrm{rad} / \mathrm{s}$

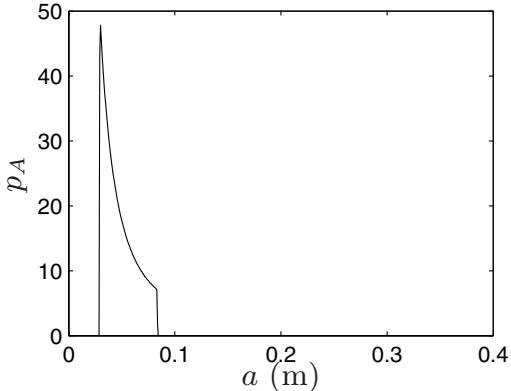

Fig. 4. Constant excitation frequency study: $A$ pdf $\left(p_{A}\right)$ for operating points (a)-(d).

(a) $\omega / \pi=1.50 \mathrm{rad} / \mathrm{s}$

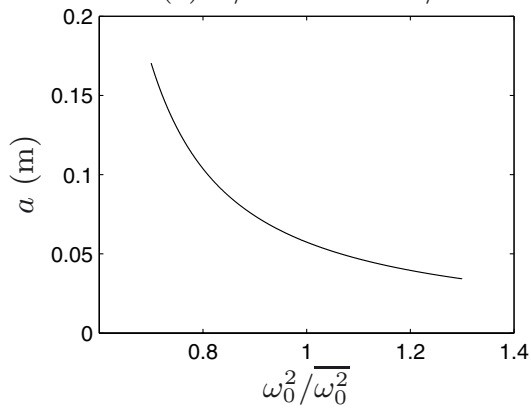

(c) $\omega / \pi=2.00 \mathrm{rad} / \mathrm{s}$

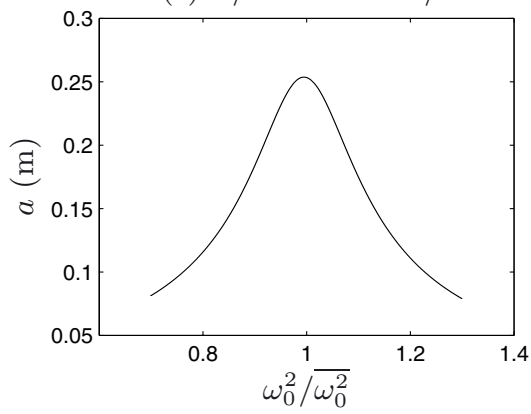

(b) $\omega / \pi=1.80 \mathrm{rad} / \mathrm{s}$

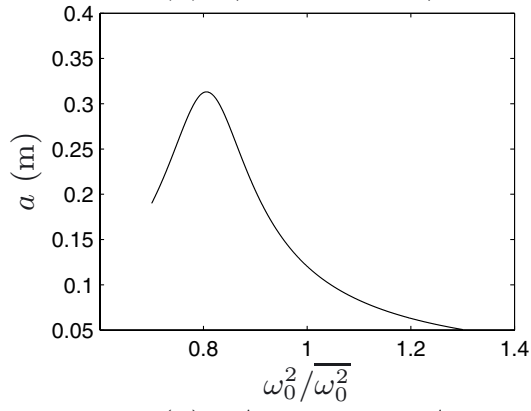

(d) $\omega / \pi=2.50 \mathrm{rad} / \mathrm{s}$

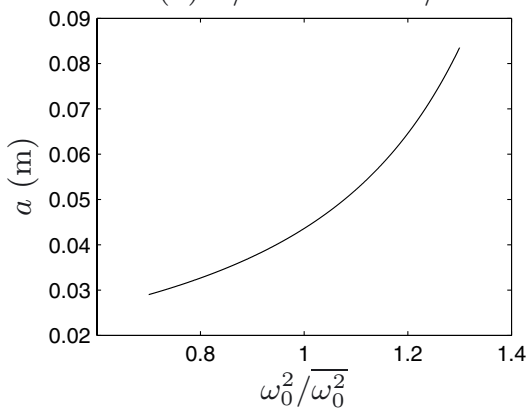

Fig. 5. Constant excitation frequency study: $a$ versus $\omega_{0}^{2}$ for operating points (a)-(d).

range $[0 ; 4 \pi]$. For each $\omega$ value, 20001 realizations of $\Omega_{0}^{2}$ are considered; these realizations are equally distributed over the range $\mathcal{I}\left(\Omega_{0}^{2}\right)$. For each $\Omega_{0}^{2}$ realization, the corresponding value for $A$ is evaluated using Equation (6) and stored. The empirical pdf $p_{A}$ is then evaluated for each excitation frequency $\omega$. Figure 3 displays $p_{A}$ for the whole $\omega$ range using colors while Figure 4 displays $p_{A}$ in a classical way for the four $\omega$ values defined by operating points $(\mathrm{a}-\mathrm{d})$ marked in Figure 2.
Panes (a) and (d) in Figure 4 show smooth pdfs whereas discontinuous pdfs similar to pane (b) curve can be observed for the excitation range $\omega \in[1.66 \pi ; 2 \pi[$ and $\omega \in] 2 \pi ; 2.28 \pi]$. The exception in this range is the pdf obtained in pane (c). Detailed explanations for these behaviors can be found in the previously mentioned references. Only the main phenomenon will be outlined here using Figure 5 which plots $a$ versus $\omega_{0}^{2}$ for the same four excitation frequencies values as in Figure 4. Continuous pdfs 
(a) $\omega / \pi=1.50 \mathrm{rad} / \mathrm{s}$

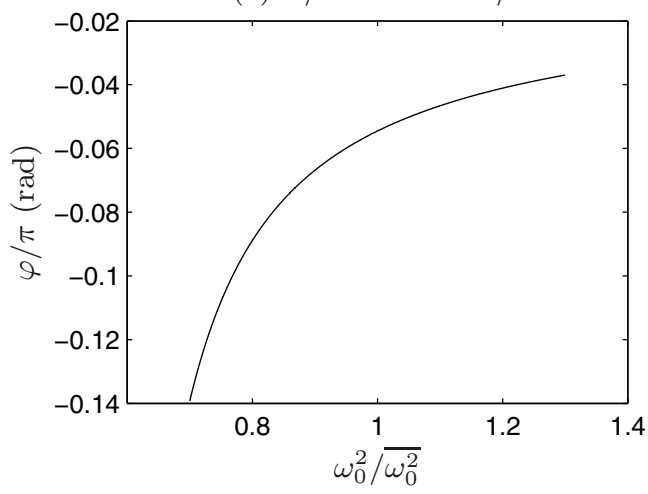

(c) $\omega / \pi=2.00 \mathrm{rad} / \mathrm{s}$

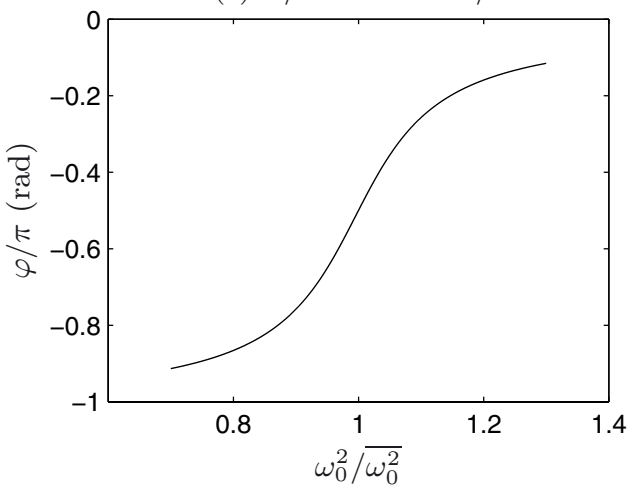

(b) $\omega / \pi=1.80 \mathrm{rad} / \mathrm{s}$

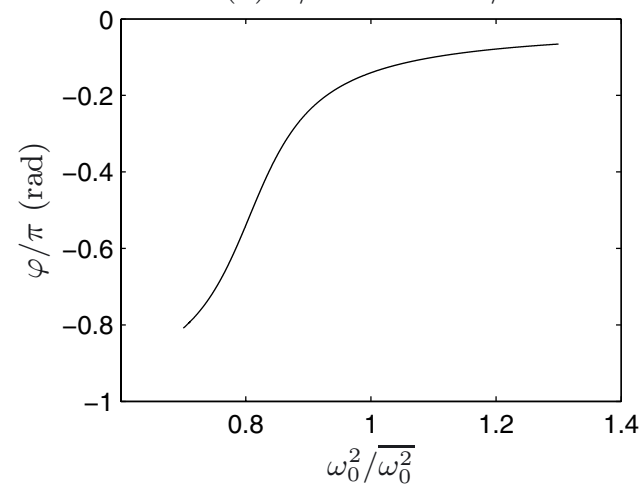

(d) $\omega / \pi=2.50 \mathrm{rad} / \mathrm{s}$

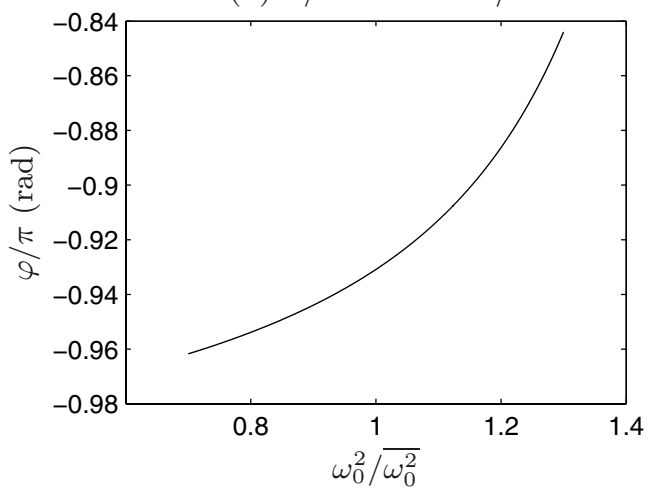

Fig. 6. Constant excitation frequency study: $\varphi$ versus $\omega_{0}^{2}$ for operating points (a)-(d).

of panes (a) and (d) in Figure 4 are related to a bijective relation between $\omega_{0}^{2}$ and $a$ as displayed by panes (a) and (d) in Figure 5. On the contrary, Figure 4b shows that different values of $\omega_{0}^{2}$ map identical values of $a$. Being represented twice, theses values for $a$ are linked to suddenly higher pdf values. The exception of Figure $3 \mathrm{c}$ comes from the symmetrical property visible in Figure $5 \mathrm{c}$ : each $a$ value has 2 preimages. Figure 6 displays plots similar to Figure 5 except that they display $\varphi$ instead of $a$ when $\omega_{0}^{2}$ varies.

These discontinuous and possibly multimodal pdfs are difficult to obtain when applying the widely used Polynomial Chaos method to approximate $A$ and $\Phi$ as illustrated further in Section 5. More complex methods must then be deployed to handle the problem [16].

\section{Consideration of a constant phase}

To avoid the previously mentioned drawbacks of using a constant excitation frequency method which can be explained by the non-bijective link between the square eigen frequency $\omega_{0}^{2}$ and the response amplitude $a$ for a given $\omega$ value, let us observe $a$ when the response phase $\varphi$ is kept constant. Equation (4) creates a link between the triplet $(a, \varphi, \omega)$; instead of choosing $\omega$ and evaluating subsequent $a$ and $\varphi$ values, let us choose a $\varphi$ value and evaluate subsequent $a$ and $\omega$ values. From a mechanical point of view, this makes sense: when $\omega_{0}^{2}$ varies, responses sharing a same phase $\varphi$ match similar operating points (maximum response amplitude for example). From a mathematical point of view, it is interesting since the link between $\omega_{0}^{2}$ and $a$ is bijective when $\varphi$ is kept constant as illustrated by Figure 9 . This figure displays graphics equivalent to Figure 5 (that is $a$ versus $\omega_{0}^{2}$ ) but for four given values of $\varphi$ instead of using given values for $\omega$. Figure 10 shows that the link between $\omega$ and $\omega_{0}^{2}$ for the considered $\varphi$ values is also bijective.

The next subsection develops the equations giving the expressions of $\omega$ and $a$ for a given $\varphi$ value. An expression for the pdf of $A$ in the case when $\Omega_{0}^{2}$ follows a uniform distribution is then derived, showing its continuity. The second subsection illustrates this constant phase method and provides graphics equivalent to those in Figures 3 to 6 .

\subsection{Expressions of $\omega, a$ and $p_{A}$ for a given $\varphi$}

For a given $\varphi \in]-\pi, 0[$ value, Equation (7) imposes $\omega$ as follows:

Case $\varphi=-\frac{\pi}{2}$ :

$$
\omega=\omega_{0}
$$

Case $\varphi \neq-\frac{\pi}{2}: \omega$ is solution of a second order polynomial equation

$$
-\tan (\varphi)^{2} \omega^{2}+2 \eta \omega_{0} \omega+\tan (\varphi)^{2} \omega_{0}^{2}=0
$$


which has two possible solutions:

$$
\omega=\frac{\omega_{0}}{\tan (\varphi)}\left(\eta \pm \sqrt{\eta^{2}+\tan (\varphi)^{2}}\right)
$$

Case $-\pi<\varphi<-\frac{\pi}{2}$ : then $\tan (\varphi)>0$,

$$
\begin{gathered}
\eta+\sqrt{\eta^{2}+\tan (\varphi)^{2}}>0 \text { and } \eta-\sqrt{\eta^{2}+\tan (\varphi)^{2}}<0 . \\
\omega=\frac{\omega_{0}}{\tan (\varphi)}\left(\eta+\sqrt{\eta^{2}+\tan (\varphi)^{2}}\right), \omega>\omega_{0}
\end{gathered}
$$

Case $-\frac{\pi}{2}<\varphi<0$ : then $\tan (\varphi)<0$,

$$
\begin{gathered}
\eta+\sqrt{\eta^{2}+\tan (\varphi)^{2}}>0 \text { and } \eta-\sqrt{\eta^{2}+\tan (\varphi)^{2}}<0 . \\
\omega=\frac{\omega_{0}}{\tan (\varphi)}\left(\eta-\sqrt{\eta^{2}+\tan (\varphi)^{2}}\right), \omega<\omega_{0}
\end{gathered}
$$

This leads to three cases for the displacement amplitude $a$ formula due to Equation (6):

$$
\begin{aligned}
\text { Case }-\pi<\varphi & <-\frac{\pi}{2}: \\
a=\frac{1}{\omega_{0}^{2}} & \frac{f_{0}}{\sqrt{1+\tan (\varphi)^{2}}} \\
& \times \frac{1}{\frac{1}{\tan (\varphi)^{2}}\left(\eta+\sqrt{\eta^{2}+\tan (\varphi)^{2}}\right)^{2}-1}
\end{aligned}
$$

Case $\varphi=-\frac{\pi}{2}$ :

$$
a=\frac{1}{\omega_{0}^{2}} \frac{f_{0}}{2 \eta}
$$

Case $-\frac{\pi}{2}<\varphi<0$ :

$$
\begin{aligned}
a=\frac{1}{\omega_{0}^{2}} & \frac{f_{0}}{\sqrt{1+\tan (\varphi)^{2}}} \\
& \times \frac{1}{1-\frac{1}{\tan (\varphi)^{2}}\left(\eta-\sqrt{\eta^{2}+\tan (\varphi)^{2}}\right)^{2}}
\end{aligned}
$$

In every case, $a$ can be rewritten

$$
a=\frac{a_{\varphi}}{\omega_{0}^{2}}
$$

where $a_{\varphi}$ is a coefficient depending on $\varphi$ but not on $\omega_{0}^{2}$. Hence, formulas for $A$ and its cdf can be derived:

$$
\begin{gathered}
A=\frac{a_{\varphi}}{\Omega_{0}^{2}} \\
\mathbf{P}_{A}(x)=1-\mathbf{P}_{\Omega_{0}^{2}}\left(\frac{a_{\varphi}}{x}\right)
\end{gathered}
$$

In the case when $\Omega_{0}^{2} \hookrightarrow \mathcal{U}\left(\overline{\omega_{0}^{2}}-\Delta \omega_{0}^{2} ; \overline{\omega_{0}^{2}}+\Delta \omega_{0}^{2}\right)$, one

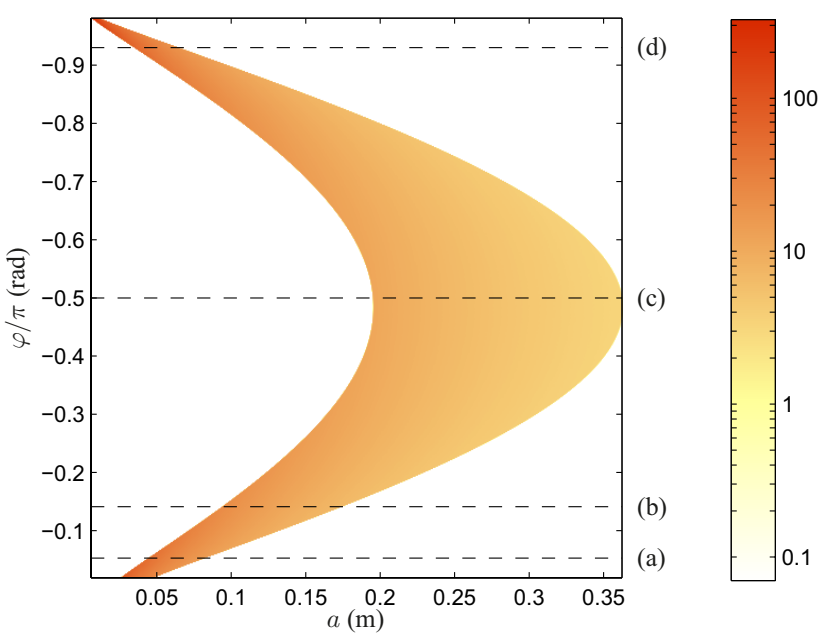

Fig. 7. Constant phase study: $A$ pdf over a given excitation frequency range. Color scale maps $\lg \left(p_{A}\right)$. (a)-(d) cuts refer to operating points defined in Figure 2.

gets:

$$
\begin{aligned}
& \mathbf{P}_{A}(x)=1+\frac{1}{2}\left(\frac{\overline{\omega_{0}^{2}}}{\Delta \omega_{0}^{2}}-1\right)-\frac{a_{\varphi}}{2 \Delta \omega_{0}^{2}} \frac{1}{x} \\
& \quad x \in\left[\frac{a_{\varphi}}{\overline{\omega_{0}^{2}}+\Delta \omega_{0}^{2}} ; \frac{a_{\varphi}}{\overline{\omega_{0}^{2}}-\Delta \omega_{0}^{2}}\right] \\
& p_{A}(x)=\frac{a_{\varphi}}{2 \Delta \omega_{0}^{2}} \frac{1}{x^{2}}, \quad x \in\left[\frac{a_{\varphi}}{\overline{\omega_{0}^{2}}+\Delta \omega_{0}^{2}} ; \frac{a_{\varphi}}{\overline{\omega_{0}^{2}}-\Delta \omega_{0}^{2}}\right]
\end{aligned}
$$

which is smooth, unlike the case when $\omega$ is kept constant.

\subsection{Numerical example}

To illustrate the constant phase method, Monte Carlo simulations similar to those of Section 3 are carried out using $501 \varphi$ values equally distributed over the range $[-0.98 \pi ;-0.02 \pi]$. For each $\varphi$ value, 20001 realizations of $\Omega_{0}^{2}$ are considered; these realizations are equally distributed over the range $\mathcal{I}\left(\Omega_{0}^{2}\right)$. For each $\Omega_{0}^{2}$ realization, the corresponding values for $\Omega$ and $A$ are evaluated using Equations (12) and (14) or Equations (9) and (15) or Equations (13) and (16) depending on $\varphi$ value. The pdf $p_{A}$ is then evaluated for each phase $\varphi$. Figure 7 displays $p_{A}$ for the whole $\varphi$ range using colors while Figure 8 displays $p_{A}$ in a classical way for the four $\varphi$ values defined by operating points $(\mathrm{a}-\mathrm{d})$ marked in Figure 2 .

All panes $(a-d)$ in Figure 8 show smooth pdfs while discontinuous pdfs were observed in the constant excitation frequency case, for $\omega \in[1.66 \pi ; 2.28 \pi]$. This is justified mathematically by Equation (21) which proves the smoothness of $p_{A}$. It can also be understood by considering the bijective link between $a$ and $\omega_{0}^{2}$ emphasized by Figure 9 which was previously mentioned. Finally let us 
E. Sarrouy et al.: Mechanics \& Industry 17, 206 (2016)

(a) $\varphi / \pi=-0.05 \mathrm{rad} / \mathrm{s}$

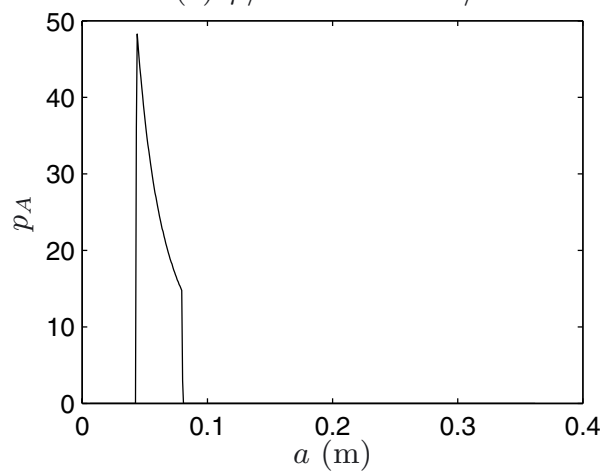

(c) $\varphi / \pi=-0.50 \mathrm{rad} / \mathrm{s}$

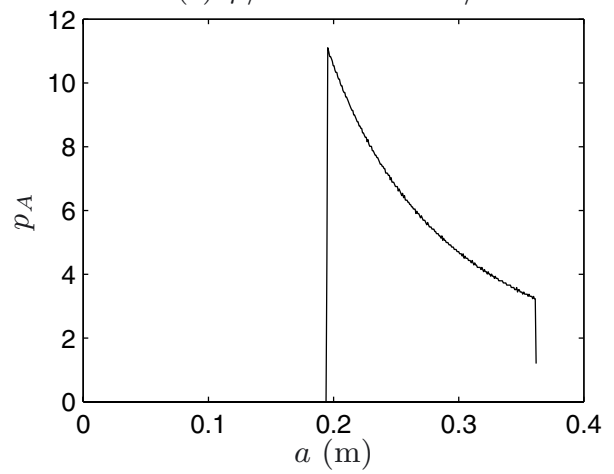

(b) $\varphi / \pi=-0.14 \mathrm{rad} / \mathrm{s}$

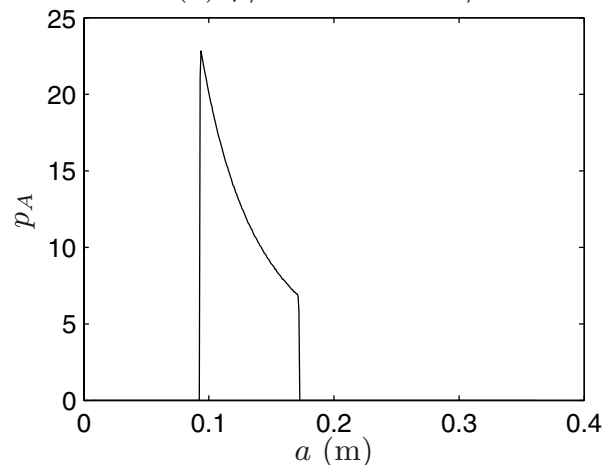

(d) $\varphi / \pi=-0.93 \mathrm{rad} / \mathrm{s}$

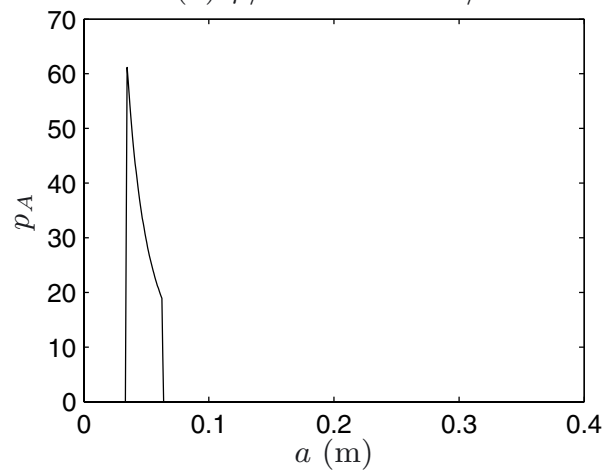

Fig. 8. Constant phase study: $A$ pdf for operating points (a)-(d).

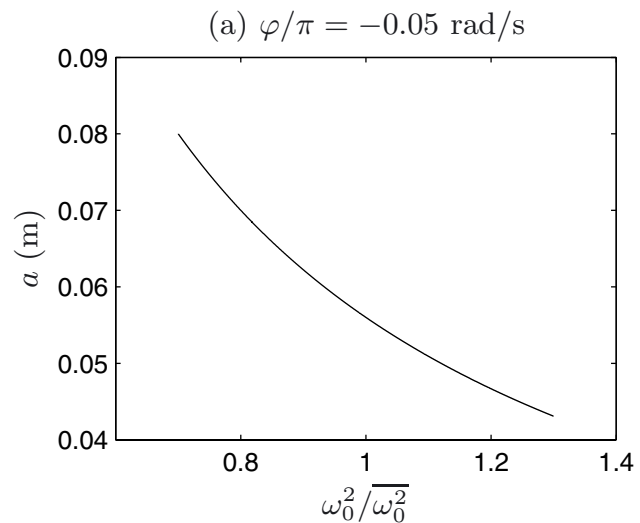

(c) $\varphi / \pi=-0.50 \mathrm{rad} / \mathrm{s}$

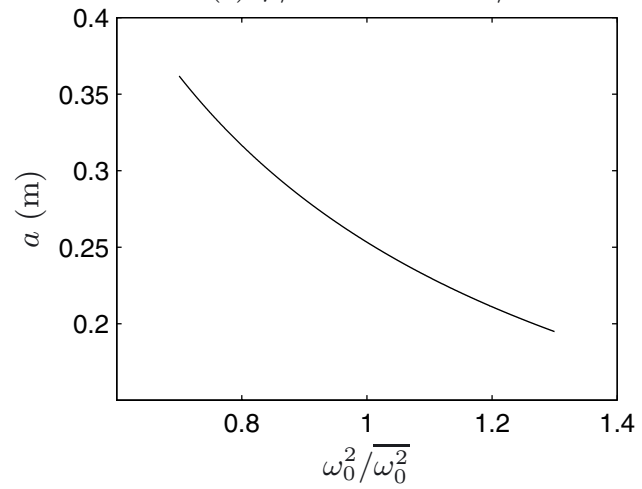

(b) $\varphi / \pi=-0.14 \mathrm{rad} / \mathrm{s}$

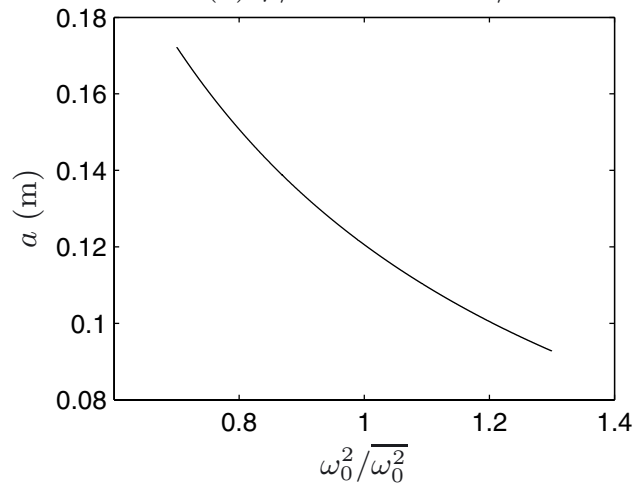

(d) $\varphi / \pi=-0.93 \mathrm{rad} / \mathrm{s}$

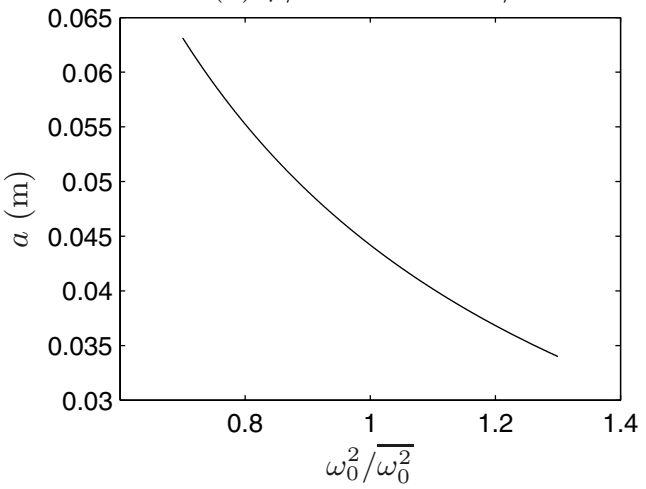

Fig. 9. Constant phase study: $a$ versus $\omega_{0}^{2}$ for operating points (a)-(d). 
(a) $\varphi / \pi=-0.05 \mathrm{rad} / \mathrm{s}$

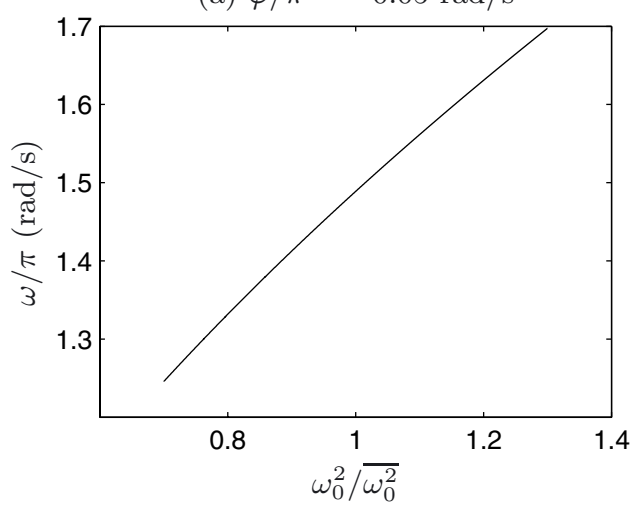

(c) $\varphi / \pi=-0.50 \mathrm{rad} / \mathrm{s}$

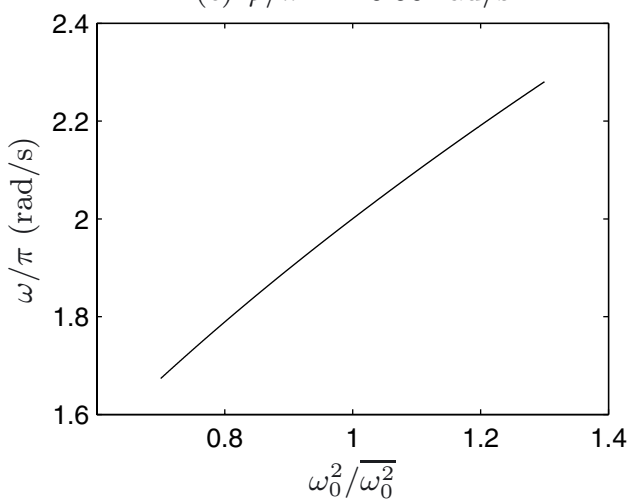

(b) $\varphi / \pi=-0.14 \mathrm{rad} / \mathrm{s}$

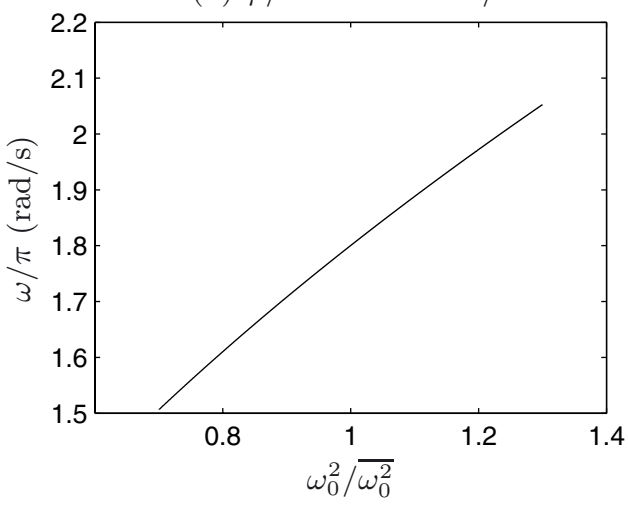

(d) $\varphi / \pi=-0.93 \mathrm{rad} / \mathrm{s}$

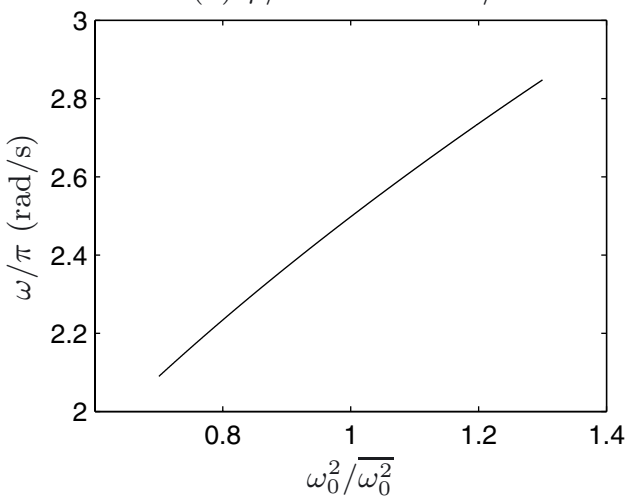

Fig. 10. Constant phase study: $\omega$ versus $\omega_{0}^{2}$ for operating points (a)-(d).

point out that the link between $\omega$ and $\omega_{0}^{2}$ is bijective too as illustrated by Figure 10 .

To figure out the main differences between the spaces involved by each method (constant excitation frequency or constant phase), Figure 11 displays the variations around operating points $(\mathrm{a}-\mathrm{d})$ when $\omega_{0}^{2}$ varies over $\mathcal{I}\left(\Omega_{0}^{2}\right)$ for both methods in classical diagrams: amplitude $a$ and phase $\varphi$ versus excitation frequency $\omega$.

\section{Consequences on a polynomial chaos study}

Previous illustrations are based on Monte Carlo simulations: the direct problem (considering either a constant excitation frequency or a constant phase) is solved for a large sample of $\Omega_{0}^{2}$ realizations. This can be afforded here because the system is very small and the cost of the direct evaluation is trivial. However, stochastic systems are often studied using an approximation of the stochastic response [17-19] in order to decrease the computational cost. Among the different methods used to compute such approximations, the Polynomial Chaos expansion (PCE) introduced by Wiener [7] and recently expanded to generalized Polynomial Chaos (gPC) expansion and MultiElement generalized Polynomial Chaos (MEgPC) expansion $[11,13,20]$ is one of the most famous.

The next subsection provides a brief description of the simple Polynomial Chaos expansion, while the second one compares the results obtained when combining each method (constant excitation frequency and constant phase) with PCE.

\subsection{Brief summary about polynomial chaos expansion}

Only the principle is recalled here for a dimension-one stochastic space, that is when only one random variable $\xi$ is used to introduce randomness in the system. The reader is referred to the references cited above for a complete presentation of PCE.

Considering a second-order random process $X$, the Polynomial Chaos expansion proposes to express it as a function $\hat{X}$ which is a polynomial series using a set of $N$ orthogonal polynomials denoted $\psi_{n}$ in the variable $\xi$ :

$$
X(\theta)=\hat{X}(\xi(\theta)):=\sum_{n=0}^{N-1} x_{n} \psi_{n}(\xi(\theta))
$$

where the order $N$ is theoretically infinite for general situations.

The deterministic coefficients $x_{n}$ are now used to represent $X$. They can be evaluated in two ways: using an intrusive method or a non-intrusive one. The intrusive method follows a Galerkin approach: Equation (22) is introduced in the equations governing $X$ and these equations are projected onto the set of orthogonal polynomials $\psi_{n}$. The non-intrusive method uses the orthogonality 
(A) Amplitude - Constant $\omega$

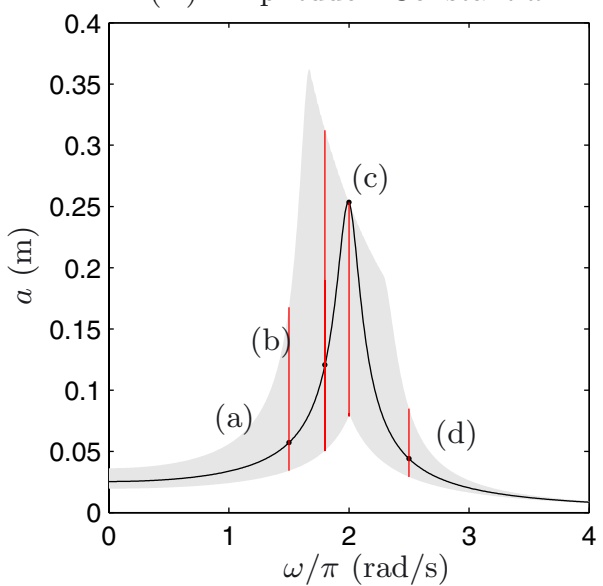

(C) Amplitude - Constant phase

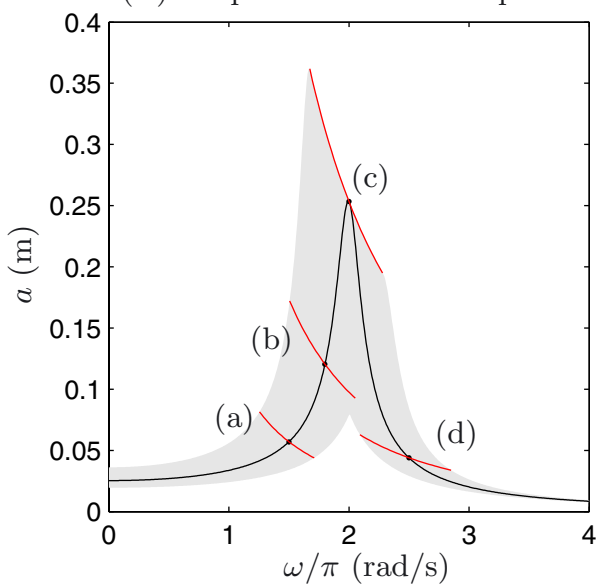

(B) Phase - Constant $\omega$

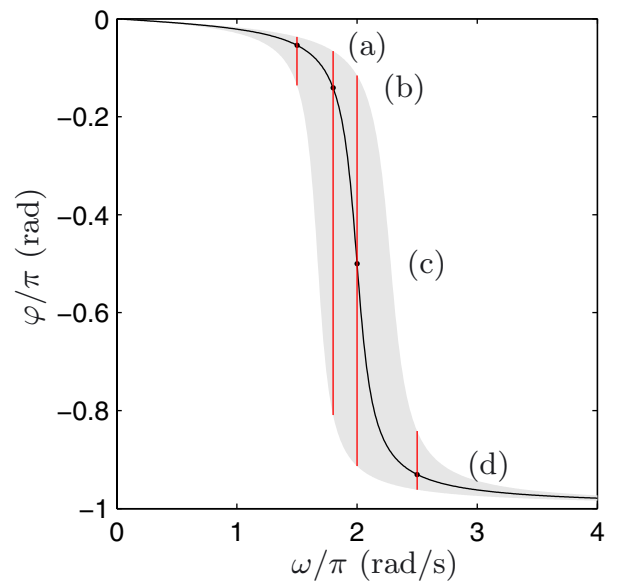

(D) Phase - Constant phase

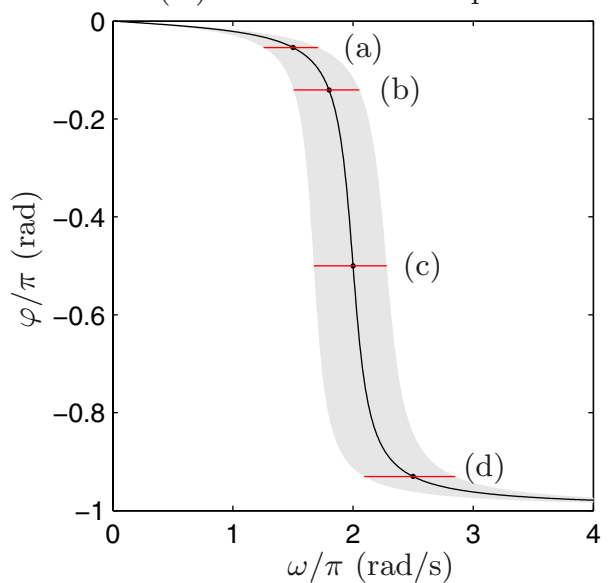

Fig. 11. Deterministic frequency response diagrams for the mean sdof damped oscillator plus variation of (A,C) amplitude and $(B, D)$ phase when $\omega_{0}^{2}$ varies around operating points $(a)-(d)$ using a $(A, B)$ constant frequency or a $(C, D)$ constant phase approach. Grey patches display the domains of possible amplitudes or phases considering the whole $\omega_{0}^{2}$ variation range.

of the polynomials with respect to a scalar product denoted $\langle\bullet, \bullet\rangle$ :

$$
x_{n}=\frac{\left\langle\hat{X}, \psi_{n}\right\rangle}{\left\langle\psi_{n}, \psi_{n}\right\rangle}
$$

where the numerator is usually evaluated using a quadrature rule.

The main difference between both methods is that the intrusive method provides a set of $m \times N$ coupled algebraic equations (where $m$ is the size of the underlying deterministic problem) and often requires a special implementation while the non-intrusive approach determines the set of coefficients $x_{n}$ one after the other in an independent manner and reuses existing codes to evaluate $X$ realizations needed for the quadrature.

The choice of the polynomial basis is somehow arbitrary even if some bases are considered as optimal to describe some distributions by some authors, as Xiu and Karniadakis [11]. In the present case, the random input $\Omega_{0}^{2}$ follows a uniform distribution which makes the
Legendre polynomial basis the most natural choice. The first 6 polynomials are:

$$
\begin{array}{llrl}
\psi_{0}(x) & =1 & \psi_{4}(x) & =\frac{1}{8}\left(35 x^{4}-30 x^{2}+3\right) \\
\psi_{1}(x) & =x & \psi_{5}(x) & =\frac{1}{8}\left(63 x^{5}-70 x^{3}+15 x\right) \\
\psi_{2}(x) & =\frac{1}{2}\left(3 x^{2}-1\right) & \psi_{6}(x) & =\frac{1}{16}\left(231 x^{6}-315 x^{4}\right. \\
\psi_{3}(x) & =\frac{1}{2}\left(5 x^{3}-3 x\right) & & \left.+105 x^{2}-5\right)
\end{array}
$$

This set of polynomials is orthogonal with respect to the following scalar product

$$
\langle f, g\rangle=\frac{1}{2} \int_{-1}^{1} f(x) g(x) \mathrm{d} x
$$

The adequacy of the Legendre polynomial basis and the expansion on a random variable $\xi$ that follows a uniform distribution $\mathcal{U}(-1 ; 1)$ and hence has $p_{\xi}(x)=\frac{1}{2}$ as probability density function may become 

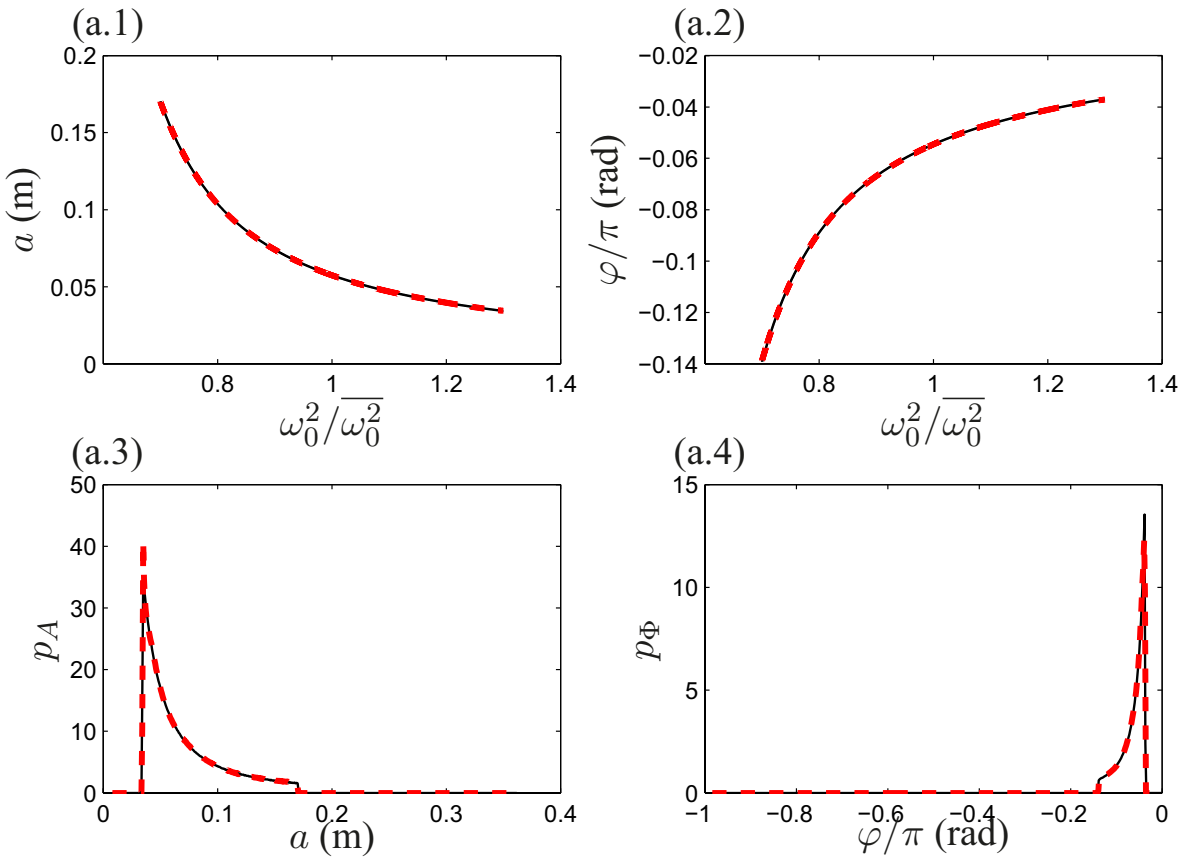

- MC simulations $\quad=--$ PC simulations

Fig. 12. Constant excitation frequency study: PC simulations compared to MC simulations for operating point (a).

visible if the numerator of Equation (23) is rewritten as follows:

$$
\begin{aligned}
\left\langle\hat{X}, \psi_{n}\right\rangle & =\int_{-1}^{1} \hat{X}(x) \psi_{n}(x) \frac{1}{2} \mathrm{~d} x \\
& =\int_{-1}^{1} \hat{X}(x) \psi_{n}(x) p_{\xi}(x) \mathrm{d} x=\mathrm{E}\left[\hat{X} \psi_{n}\right]
\end{aligned}
$$

where $\mathrm{E}[X]$ denotes the expected value of random variable $X$.

Once PCE coefficients $x_{n}$ are evaluated, there are two ways to post-process them. First, the mean and variance can be directly computed provided $\psi_{0}(x)=1$ :

$$
\begin{aligned}
& \mathrm{E}[\hat{X}]=x_{0}\left\langle\psi_{0}, \psi_{0}\right\rangle \\
& \text { and } \mathrm{E}\left[(\hat{X}-\mathrm{E}[\hat{X}])^{2}\right]=\sum_{n=1}^{N-1} x_{n}^{2}\left\langle\psi_{n}, \psi_{n}\right\rangle
\end{aligned}
$$

Second, cdf and pdf can be evaluated based on MC simulations. The difference with the usual processing is that $\hat{X}$ realizations are computed using its PCE (i.e. Eq. (22)) rather than solving the direct problem which saves a lot of computational time and resource when the samples are large.

\subsection{Application of PCE for both methods}

Let us define the random variable $\xi$ as follows:

$$
\xi=\frac{\Omega_{0}^{2}-\overline{\omega_{0}^{2}}}{\Delta \omega_{0}^{2}}
$$

Its cdf $\mathbf{P}_{\xi}$ and pdf $p_{\xi}$ can be easily established:

$$
\mathbf{P}_{\xi}(x)=\frac{x+1}{2}, p_{\xi}(x)=\frac{1}{2}, x \in[-1,1]
$$

It follows that $\xi$ has a uniform distribution $\mathcal{U}(-1 ;+1)$. This random variable will serve to develop all the stochastic quantities around the 4 operating points $(\mathrm{a}-\mathrm{d})$ defined in Figure 2 and which correspond to the 4 following triplets:

$$
\begin{aligned}
& \left(\omega_{a}^{\mathrm{op}}, a_{a}^{\mathrm{op}}, \varphi_{a}^{\mathrm{op}}\right)=(1.5 \pi, 0.0571,-0.0540 \pi) \\
& \left(\omega_{b}^{\mathrm{op}}, a_{b}^{\mathrm{op}}, \varphi_{b}^{\mathrm{op}}\right)=(1.8 \pi, 0.1205,-0.1408 \pi) \\
& \left(\omega_{c}^{\mathrm{op}}, a_{c}^{\mathrm{op}}, \varphi_{c}^{\mathrm{op}}\right)=(2.0 \pi, 0.2533,-0.5000 \pi) \\
& \left(\omega_{d}^{\mathrm{op}}, a_{d}^{\mathrm{op}}, \varphi_{d}^{\mathrm{op}}\right)=(2.5 \pi, 0.0440,-0.9304 \pi)
\end{aligned}
$$

When the constant excitation frequency method is applied, $\omega$ is set to $\omega_{x}^{\text {op }}(x \in\{a, b, c, d\})$ and $A$ and $\Phi$ expansions are evaluated. In the case when the constant phase method is applied, $\varphi$ is set to $\varphi_{x}^{\text {op }}(x \in\{a, b, c, d\})$ and $A$ and $\Omega$ expansions are evaluated. A degree 6 expansion is used in every case. The coefficients are evaluated using a non-intrusive method relying on a Gauss-Legendre quadrature with 7 nodes.

Figures 12 to 15 provide comparisons of Monte Carlo simulations and PCE results around operating points (a-d) when the constant excitation frequency method is used. As expected, this method provides correct result for operating points (a), Figure 12 and (d), Figure 15: variations of $a$ and $\varphi$ with $\omega_{0}^{2}$ are well reproduced by the $\mathrm{PCE}$ and so are the respective pdfs. However, PCE does 
E. Sarrouy et al.: Mechanics \& Industry 17, 206 (2016)
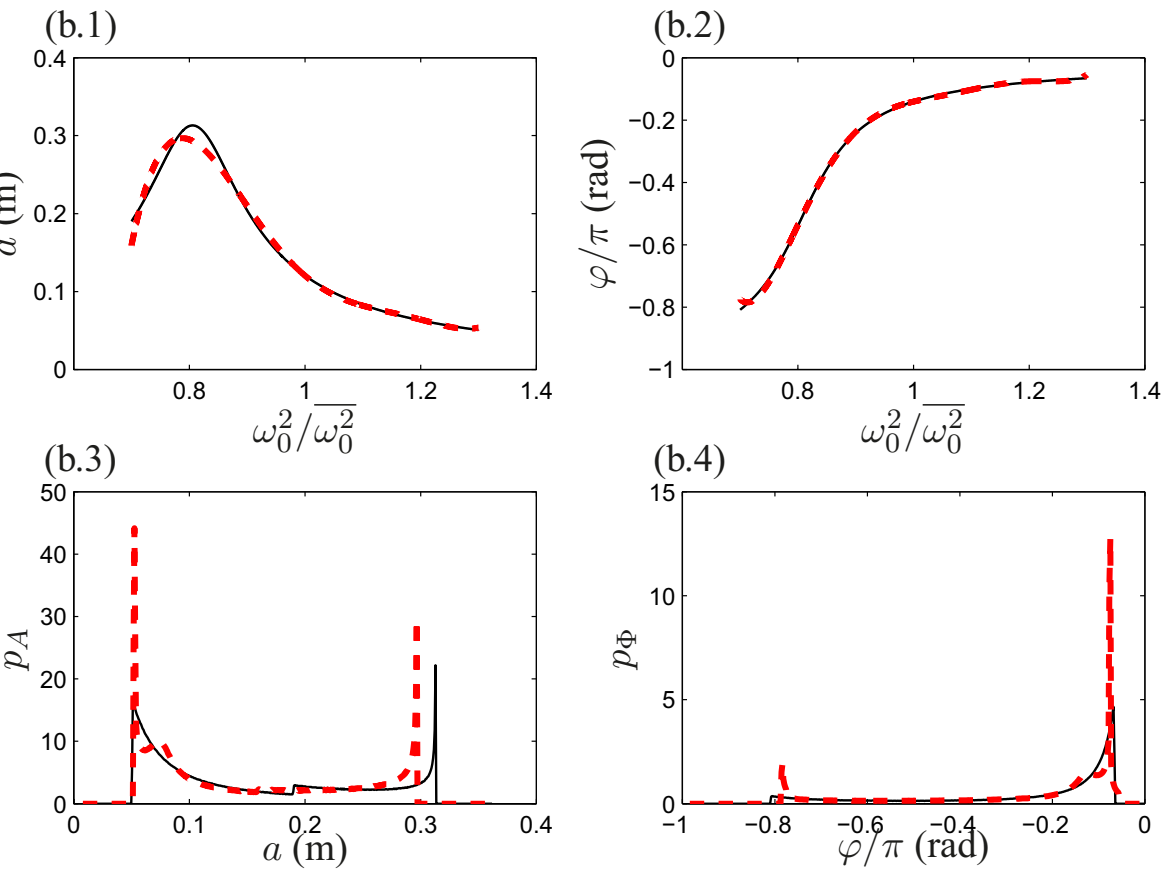

- MC simulations

- - - PC simulations

Fig. 13. Constant excitation frequency study: PC simulations compared to MC simulations for operating point (b).
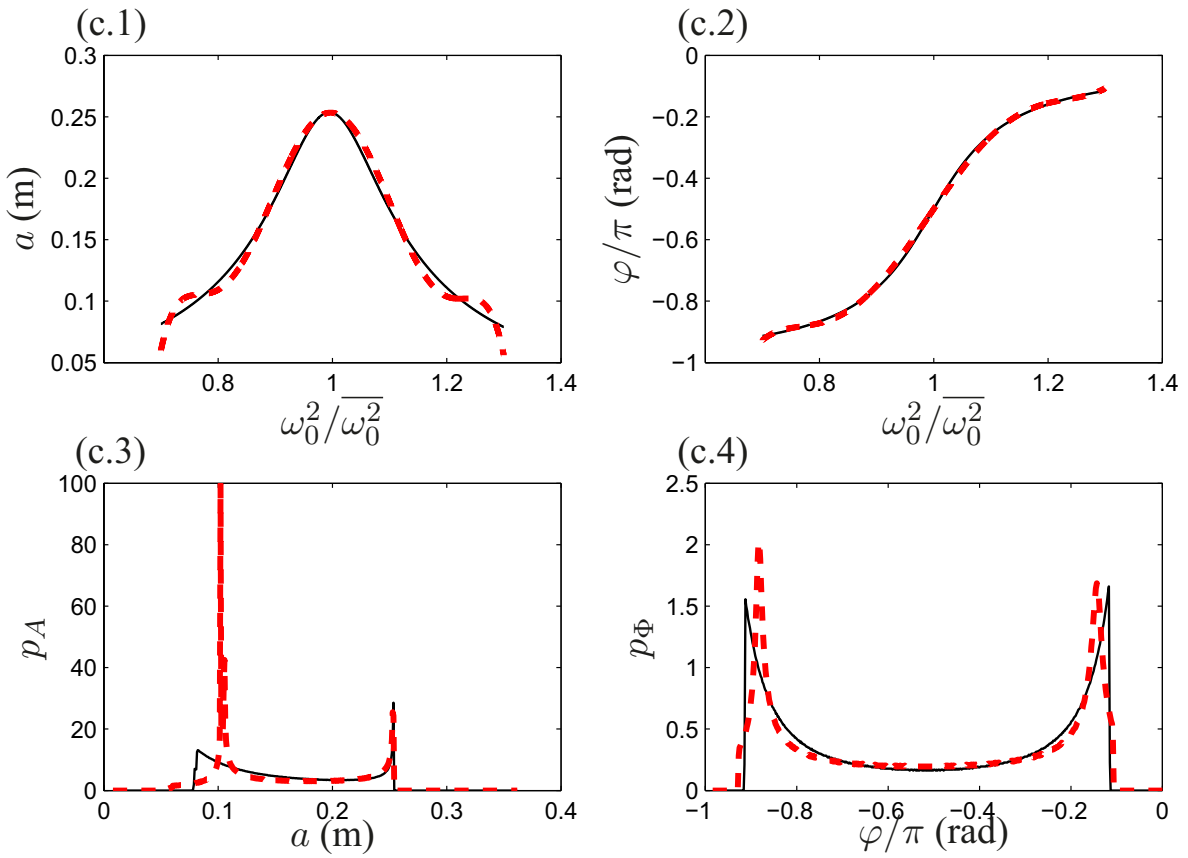

— MC simulations _ = - PC simulations

Fig. 14. Constant excitation frequency study: PC simulations compared to MC simulations for operating point (c). 

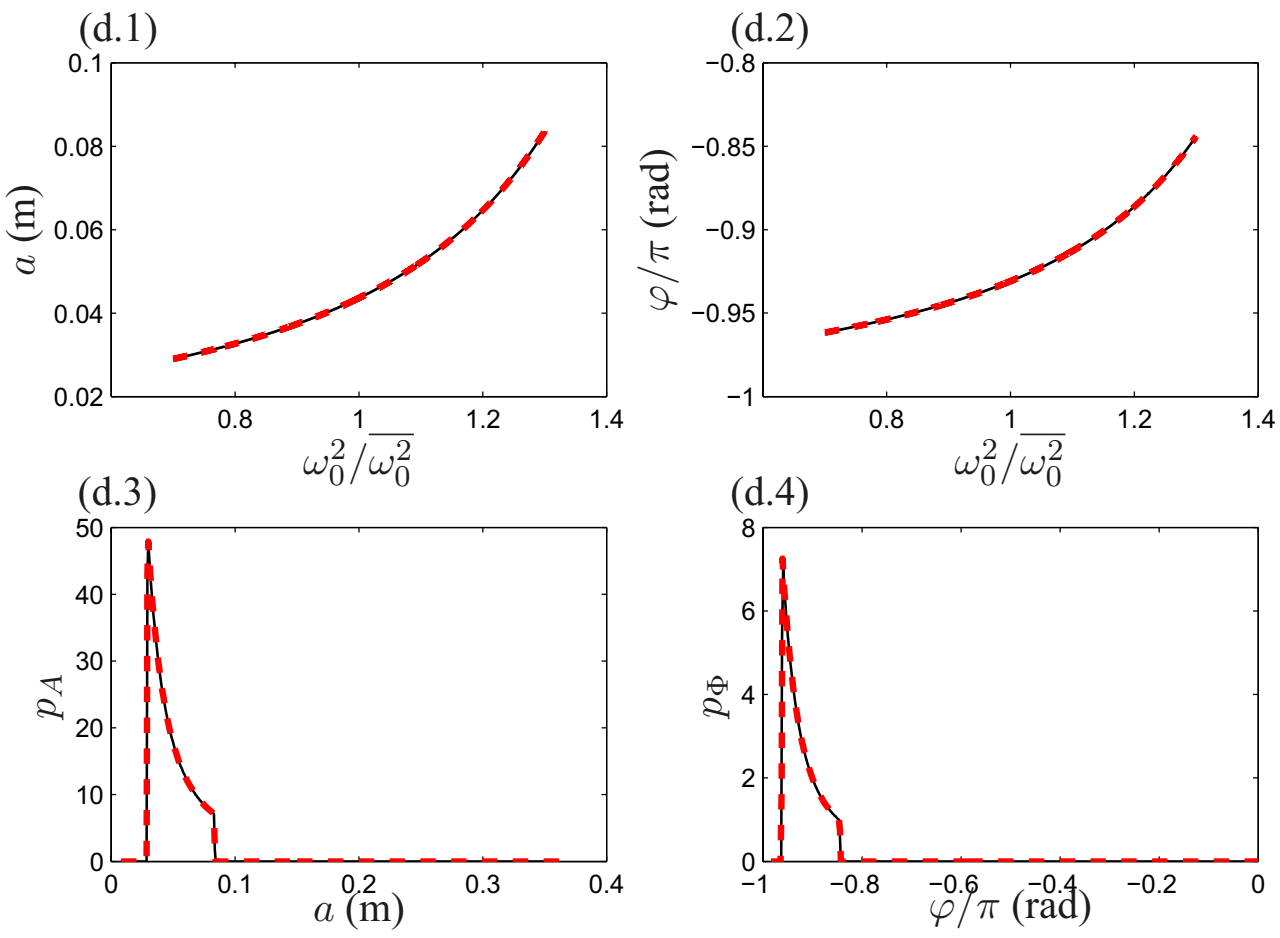

- MC simulations - - - PC simulations

Fig. 15. Constant excitation frequency study: PC simulations compared to MC simulations for operating point (d).

not provide a proper description of $A$ and $\Phi$ for operating points (b), Figure 13 and (c), Figure 14. As stated in [16], increasing the expansion degree would not provide better results.

Figures 16 to 19 provide comparisons of Monte Carlo simulations and PCE results around operating points (a-d) when the constant phase method is used. In this case, the variation of $a$ and $\omega$ with $\omega_{0}^{2}$ is well described by the PCE as well as the corresponding pdf, both being much smoother than when the constant excitation frequency is used.

\subsection{Comments on the practical use of the constant phase approach}

The constant phase approach is obviously useful when one wants to study the variation of the system response in a particular configuration which is characterized by the phase $\varphi$ : variation of the resonance peak $(\varphi=$ $\left.\arctan \left(-\sqrt{1-2 \eta^{2}} / \eta\right)\right)$, variation of the system response when in quadrature with the excitation $(\varphi=-\pi / 2), \ldots$

However, one frequently wants to check that the system response will not exceed some given values over a range of excitation frequency. In this case, the constant excitation frequency approach seems more adapted but returns erroneous results for some system parameters when using PCE (see Pagnacco et al. $[6,16]$ ), especially around resonance that is where amplitudes are gener- ally controlled. The constant phase approach can still be used if combined to a little post processing: let us denote $\left[f_{\min } ; f_{\max }\right]$ the frequency range of interest. Evaluation of the response of the mean system for $f_{\min }$ and $f_{\max }$ provides coarse upper and lower bounds for the phase response. As depicted in Figure 11, it is necessary to enlarge this range to properly cover the whole frequency range $\left[f_{\min } ; f_{\max }\right]$. By enlarging this phase range, one gets the systems stochastic response over the desired frequency range. Depending on the desired statistical indicators (confidence interval, quantiles, moments, ...), an adapted post-processing can be implemented.

This said, it is nonetheless interesting to keep in mind that being able to describe the variation of the resonance peak both in terms of amplitude and frequency is much more interesting that knowing that the amplitude may stay below a given value over $f_{\min }$ and $f_{\max }$ and ignoring it will explode for a little lower excitation frequency.

\section{Conclusion}

An original approach to study the dynamic response of a single degree of freedom system has been developed. This approach proposes to expand the system response on the Polynomial Chaos when imposing the response phase and freeing the system excitation frequency rather than the other way around as it is usually done. The proposed approach was applied to a single dof system 

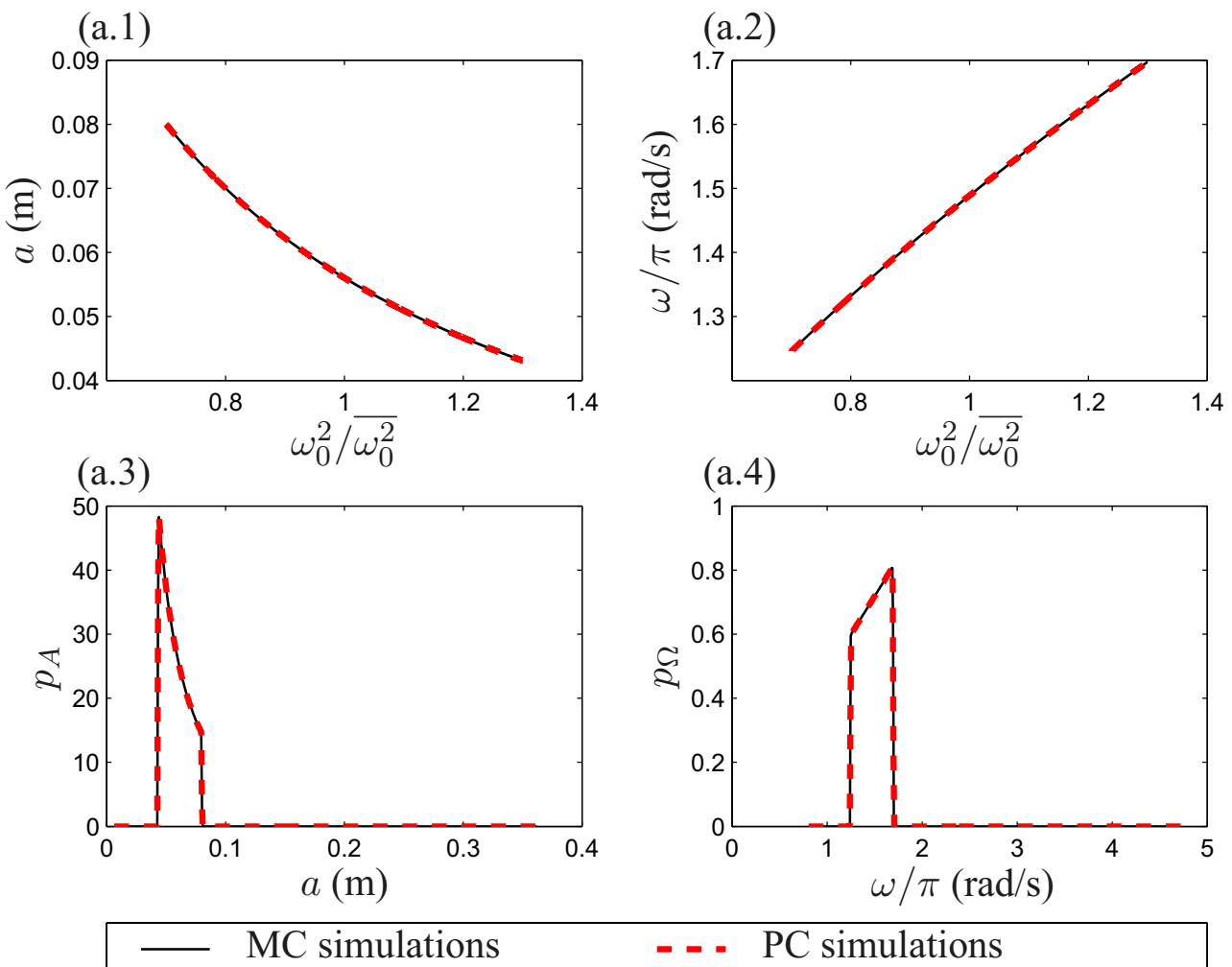

- - - PC simulations

Fig. 16. Constant phase study: PC simulations compared to MC simulations for operating point (a).
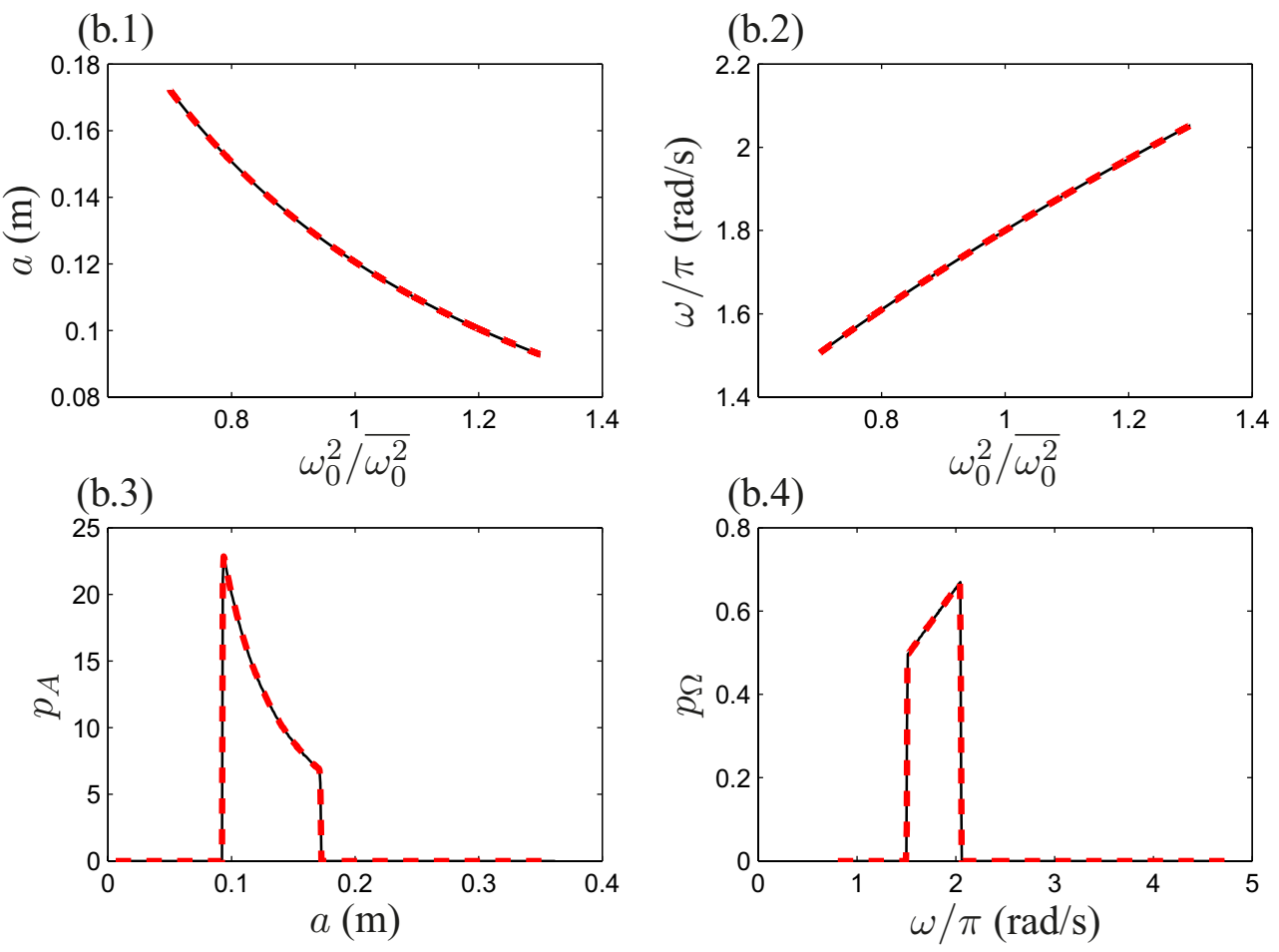

\section{— MC simulations}

- - - PC simulations

Fig. 17. Constant phase study: PC simulations compared to MC simulations for operating point (b). 

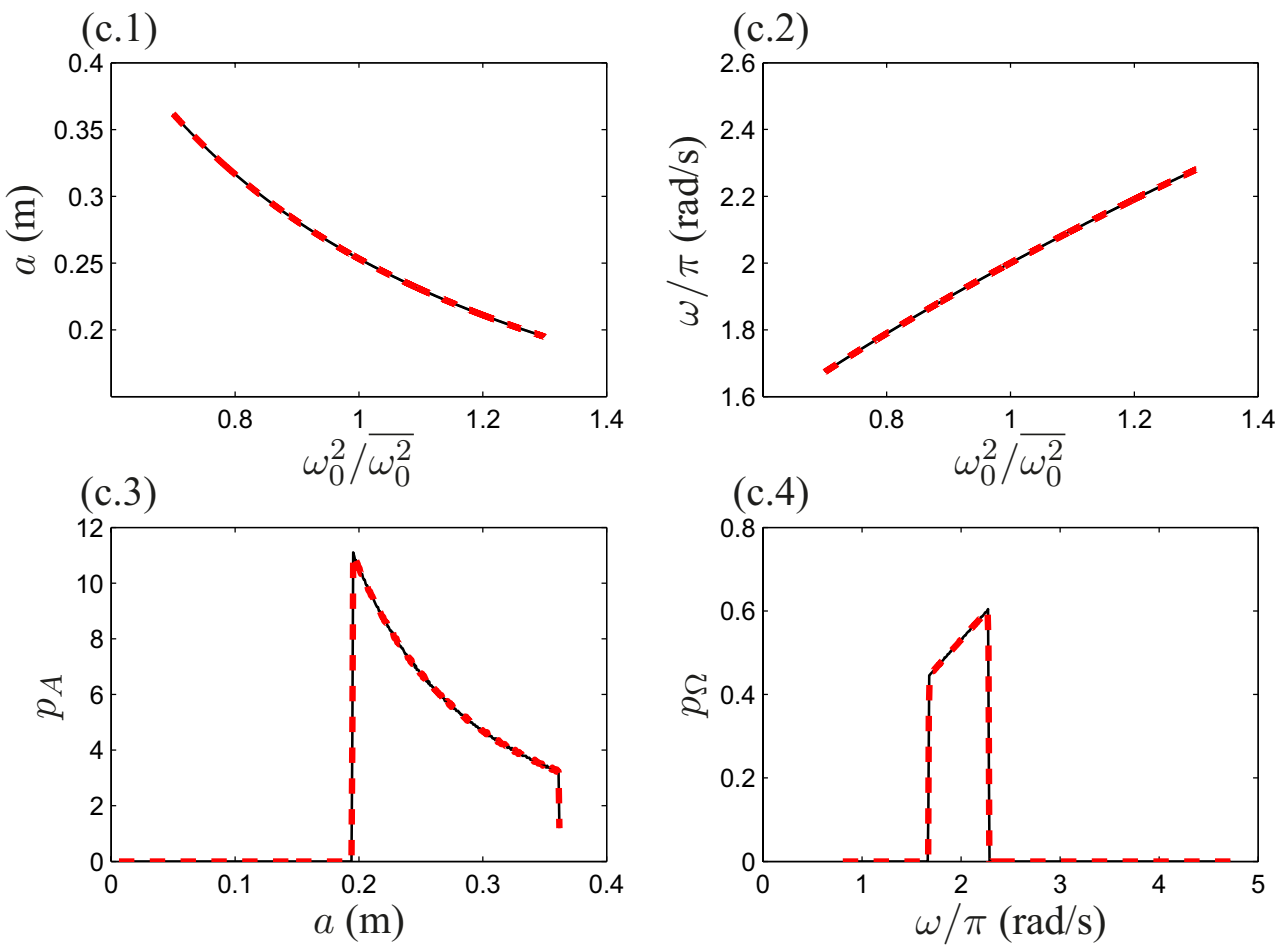

— MC simulations

- - - PC simulations

Fig. 18. Constant phase study: PC simulations compared to MC simulations for operating point (c).
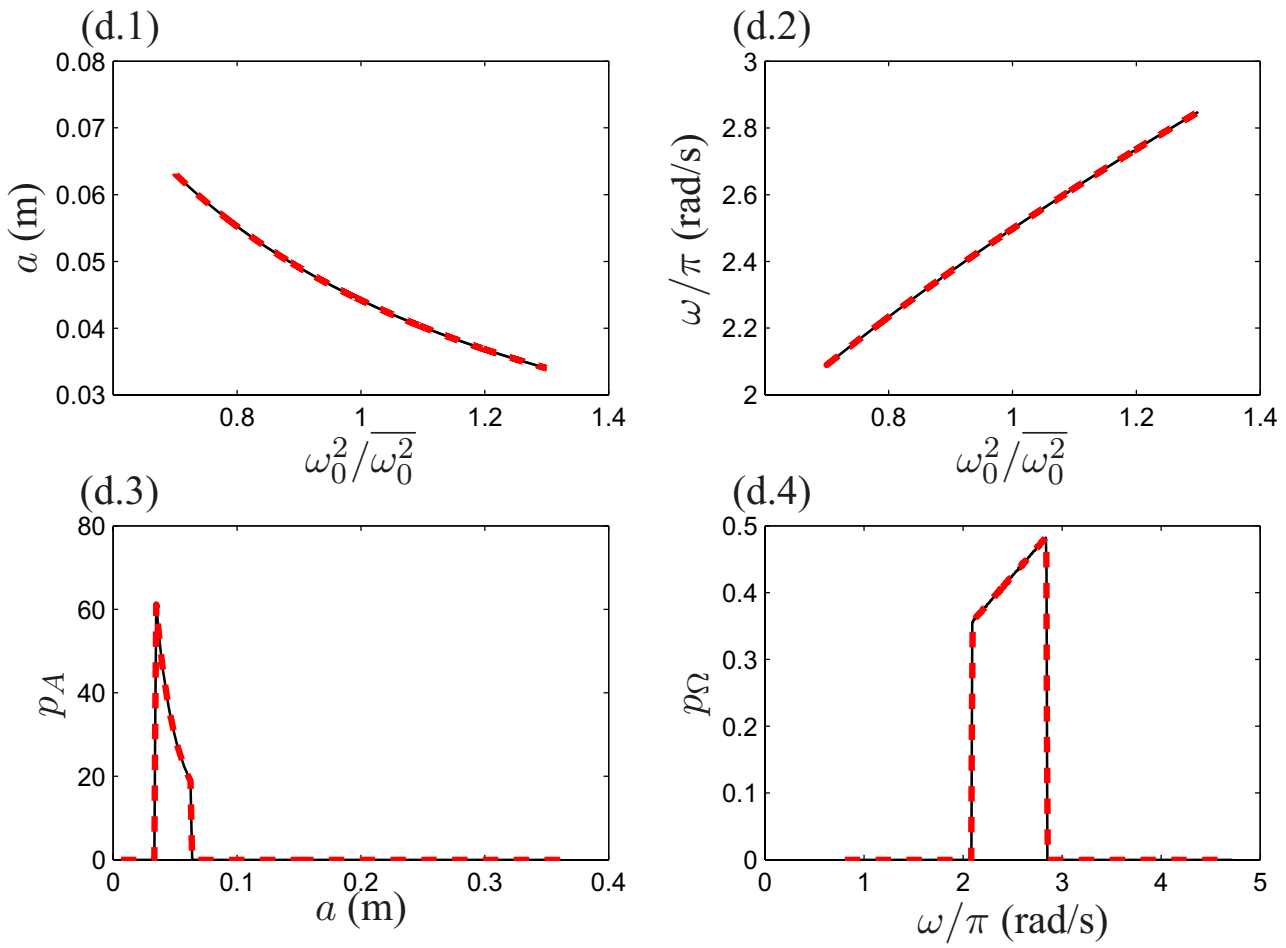

\section{— MC simulations}

- - - PC simulations

Fig. 19. Constant phase study: PC simulations compared to MC simulations for operating point (d). 
whose squared eigen frequency follows a uniform law. This method was proven to provide much better results than the usual approach which suffers from the inability to describe the multimodality of the stochastic response. The numerical application also demonstrated its ability to follow some phase defined points such as the response of the system when in quadrature with the excitation which is usually close to the resonance point and is easy to detect experimentally.

This work addressed a single dof system: further work should handle the case of multi-dofs systems for which multiple phases (one per dof) exist.

\section{References}

[1] F.E. Udwadia, Response of uncertain dynamic systems. i, Appl. Math. Comput. 22 (1987) 115-150

[2] F.E. Udwadia, Response of uncertain dynamic systems. ii, Appl. Math. Comput. 22 (1987) 151-187

[3] C. Heinkelé, S. Pernot, F. Sgard, C.-H. Lamarque, Vibration of an oscillator with random damping: Analytical expression for the probability density function, J. Sound Vib. 296 (2006) 383-400

[4] C. Heinkelé, Synthèse modale probabiliste - Théorie et applications, Ph.D. thesis, Ecole Centrale de Lyon, Université Claude Bernard-Lyon I-INSA Lyon, 2008

[5] E. Pagnacco, R. Sampaio, E. Souza de Cursi, Multimodality of the frequency response functions of random linear mechanical systems, in: Proceedings of CILAMCE Iberian-Latin-American Congress on Computational Methods in Engineering, Rio de Janeiro, Brasil, 2009.

[6] E. Pagnacco, R. Sampaio, E. Souza de Cursi, Frequency response functions of random linear mechanical systems and propagation of uncertainties, Mecánica Computacional XXX (2011) 3359-3380

[7] N. Wiener, The homogeneous chaos, Am. J. Math. 60 (1938) 897-936

[8] S.R. Winterstein, Moment-based Hermite models of random vibration, Technical Report 219, Dept. of Structural Engineering, Technical University of Denmark,
Lyngby, Denmark, 1987, available at: http://sites. google.com/site/stevewinterstein/publications-1/ archival-publications/papers-file-repository/ papers-nonlinear-and-moment-based-load-models/ Hermite_Winterstein_87.pdf?attredirects $=0 \& d=1$.

[9] S.R. Winterstein, Nonlinear vibration models for extremes and fatigue, J. Eng. Mech. 114 (1988)

[10] R.G. Ghanem, Pol D. Spanos, Stochastic Finite Elements: A Spectral Approach, Springer-Verlag, 1991

[11] D. Xiu, G.E. Karniadakis, The Wiener-Askey polynomial chaos for stochastic differential equations, SIAM J. Sci. Comput. 24 (2002) 619-644

[12] O.G. Ernst, A. Mugler, H.-J. Starkloff, E. Ullmann, On the convergence of generalized polynomial chaos expansions, ESAIM: Math. Modell. Numer. Anal. 46 (2012) 317-339

[13] X. Wan, G.E. Karniadakis, An adaptive multi-element generalized polynomial chaos method for stochastic differential equations, J. Comput. Phys. 209 (2005) 617-642

[14] X. Wan, G.E. Karniadakis, Beyond Wiener-Askey expansions: Handling arbitrary pdfs, J. Sci. Comput. 27 (2006) 455-464

[15] J. Foo, X. Wan, G.E. Karniadakis, The multi-element probabilistic collocation method (me-pcm): Error analysis and applications, J. Comput. Phys. 227 (2008) 9572-9595

[16] E. Pagnacco, E. Sarrouy, R. Sampaio, E. Souza de Cursi, Polynomial chaos for modeling multimodal dynamical systems - investigations on a single degree of freedom system, Mecánica Computacional XXXII (2013) 705-727

[17] D. Lucor, G.E. Karniadakis, Adaptive generalized polynomial chaos for nonlinear random oscillators, SIAM J. Sci. Comput. 26 (2004) 720-735

[18] S. Finette, A stochastic representation of environmental uncertainty and its coupling to acoustic wave propagation in ocean waveguides, J. Acoust. Soc. Am. 120 (2006) $2567-2579$

[19] E. Sarrouy, O. Dessombz, J-J. Sinou, Stochastic study of a non-linear self-excited system with friction, Eur. J. Mech. A 40 (2013) 1-10

[20] D. Xiu, G.E. Karniadakis, Modeling uncertainty in flow simulations via generalized polynomial chaos, J. Comput.l Phys. 187 (2003) 137-167 